\title{
Stability and Leakage Analysis of a Novel PP Based 9T SRAM Cell Using N Curve at Deep Submicron Technology for Multimedia Applications
}

\author{
Shilpi Birla ${ }^{1^{*}}$, Rakesh Kumar Singh ${ }^{2}$, Manisha Pattanaik ${ }^{3}$ \\ ${ }^{1}$ Department of Electronics and Communication (ECE), Sir Padampat Singhania University (SPSU), Udaipur, India \\ ${ }^{2}$ Department of Electronics and Communication (ECE), Bipin Tripathi Kumaon Institute of \\ Technology (BTKIT), Dwarahat Almora, India \\ ${ }^{3}$ VLSI Group, Atal Bihari Vajpayee Indian Institute of \\ Information Technology \& Management (ABV-IIITM), Gwalior, India \\ E-mail: ${ }^{*}$ shilpibirla@gmail.com
}

Received July 22, 2011; revised August 10, 2011; accepted August 17, 2011

\begin{abstract}
Due to continuous scaling of CMOS, stability is a prime concerned for CMOS SRAM memory cells. As scaling will increase the packing density but at the same time it is affecting the stability which leads to write failures and read disturbs of the conventional 6T SRAM cell. To increase the stability of the cell various SRAM cell topologies has been introduced, 8T SRAM is one of them but it has its limitation like read disturbance. In this paper we have analyzed a novel PP based 9T SRAM at $45 \mathrm{~nm}$ technology. Cell which has $33 \%$ increased SVNM (Static Voltage Noise Margin) from 6T and also 22\% reduced leakage power. N curve analysis has been done to find the various stability factors. As compared to the 10T SRAM cell it is more area efficient.
\end{abstract}

Keywords: N Curve, Scaling, SVNM (Static Voltage Noise Margin), Leakage Power, 9T SRAM Cell

\section{Introduction}

The high demand of increasing packaging density and low power SRAMs for multimedia applications leads to the problem of data stability. As ultra low power supply voltages suppresses power consumption, gate leakage and stand by current which results in increase of life time of battery. Various Read \& Write assist methods were introduced to enhance the write margin and read stability of $6 \mathrm{~T}$ Cells. Some of the techniques are CVDD (Cell Vdd) adjustment, CVSS (Cell virtual ground), dual rail power supply, negative bitline etc. But still the voltage of the conventional 6T SRAM cannot be reduced beyond $0.6 \mathrm{~V}$ for successful operation. Various topologies of SRAM cell has been introduced, 7T SRAM cell in which a read static noise margin is achieved by cutting off a pull down path during read operation but has limited write capability due to single end write operations [1]. 8T SRAM cell which is one of the popular topology which increases the stability but has its own limitation. In this paper the limitation of $8 \mathrm{~T}$ has been removed and alternative topologies have been discussed to increase the stability [2]. Although other 9T SRAM cell as in [3] is also been discussed but it suffers from read disturbance. As far as best of my knowledge this cell has not been reported yet.

In section II various factors of SRAM functional Margins has been reported. In section III the novel PP based 9T SRAM cell has been explained in detail. In section IV $\mathrm{N}$ curve has been discussed. In section $\mathrm{V}$ the analysis of various stability parameters with respect to Vdd and temperature has been discussed. In section VI the leakage power of the proposed cell has been discussed and in the end conclusion.

\section{SRAM Functional Margins}

SRAM functional margins are determined by three SRAM design Parameters: static noise margin (SNM), write margin (WRM), and cell current (Icell). Since all of them strongly depend on operating voltage (VDD), transistor channel length (Lg), and width (Wg). So the cell stability depends on the amount of $\mathrm{V}_{\mathrm{T}}$ mismatch caused by the random variation $\left(\sigma \mathrm{V}_{\mathrm{T}}\right)$ of threshold voltage $\mathrm{V}_{\mathrm{T}}$ 
and operation voltage VDD as well as cell ratios: $\gamma$-ratio for write and $\beta$-ratio for read [4].

\subsection{Static Noise Margin}

The static noise margin (SNM) is the maximum amount of noise voltage $\mathrm{VN}$ that can be tolerated at the both inputs of the cross-coupled inverters in different directions while inverters still maintain bi-stable operating points and cell retains its data [5]. In other words, the static noise margin (SNM) quantifies the amount of noise voltage VN required at the storage nodes of SRAM to flip the cell data. The cell becomes more vulnerable to noise during a read access since the " 0 " storage node rises to a voltage higher than ground (GND) due to a voltage division along the Pass gate transistors and inverter Pulldown devices between the pre-charged BL and the GND terminal of the cell. The ratio of the transistor width of Pull-down to Passgate, commonly referred to as the $\beta$-ratio determines how high the " 0 " storage node rises during a read access [4] as shown in Figure 1 for conventional 6T SRAM cell. Due to the scaling of the device to nanometer regime, the variation of $\beta$-ratio is significantly increased. This is the primary reason for increasing SNM challenge in nanometer-scale SRAM. The ratio of inverter pull-down transistors (M1 or M2) and pull-up transistors (M3 or M4) also directly impacts the cell immunity to noise. Weaker pull-up due to the variations makes the cell easier to flip as lowering its trip point of inverter, making the cell more vulnerable to noise.

When the WL is off, the SNM becomes larger than that for read access because of no rising of " 0 ” storage node from GND level [4]. The two kinds of SNMs for data retention and read access are referred to as "hold SNM margin” and "read SNM margin” [6].

\subsection{Write Margin}

The cell data is written by forcing the BL pair to the differential levels of " 1 " and " 0 " while WL is asserted to allow pass gate transistors (M5 or M6) connected to the $\mathrm{BL}$. The potential of the corresponding storage node is pulled down to the critical level that is dependent on the ratio of transistor strengths between M5 and M3 (or M6 and M4). This ratio is referred to as $\gamma$-ratio. In order to ensure robust write operation, the critical level has to be lowered than the trip point of connected inverter before the level of " 0 " written $\mathrm{BL}$ is reached to the end-point (e.g., GND). The write margin (WRM) is defined as the rest of potential difference between the BL level at which the data is flipped and the end-point (e.g., GND) as shown in Figure 1. If the cell data is flipped when the $\mathrm{BL}$ comes at $\mathrm{X} \mathrm{mV}$, where $\mathrm{X} \mathrm{mV}$ is allowed to reach to the GND level, WRM is defined as $\mathrm{X} \mathrm{mV}$. As the device sizes of Pass gate and Pull-up are scaled down to nanometer regime, the variation of $\gamma$-ratio is significantly increased. That is the reason why WRM has become just as difficult as read in nanometer-scale SRAM [7].

\subsection{Cell Current (Icell)}

The BL discharging time takes a large percentage of the total access time. The discharging time (TBL) depends on the BL capacitance, the cell current, and the required BL discharging level (VSEN). The amount of cell current (Icell) is determined by the strength of passgate and pull-down connected in series between the BL and GND as shown in Figure 1. The higher VT settings for pass gate, pull-down, and pull-up transistors in SRAM can suppress the sub-threshold leakage but it causes not only the reduction of Icell but also increases its variation [5].

\section{Proposed Novel PP Based 9T Cell}

In this paper we have proposed a novel PP based 9T SRAM Cell Figure 2. In this cell one extra signal RWL is used during read operation, during read operation we keep it at gnd voltage otherwise the value remains high. The true storage nodes are separated from the two virtual storage nodes connected between the stacked PMOS. If we look at the figure we will find that there is one extra NMOS transistor is used which creates a discharging path. It is connected to the RWL. The discharging path is used such that to discharge a precharged high bitline during the read operation.

This circuit has certain advantages like it does not have read problem as the discharging path is isolated from the true storage nodes. The write ability is also not disturbed in this structure. We have used a single wordline for both the operations read and write .This cell has better stability and it is power efficient.

\subsection{Detailed Structure of the Cell}

In this section, we describe our cell design in Figure 2. As mentioned previously, it is composed of two cross coupled P-P-N inverters, and data is stored in node Q and

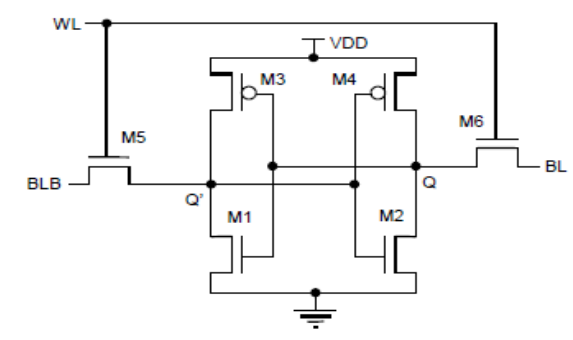

Figure 1. Conventional 6T SRAM cell. 


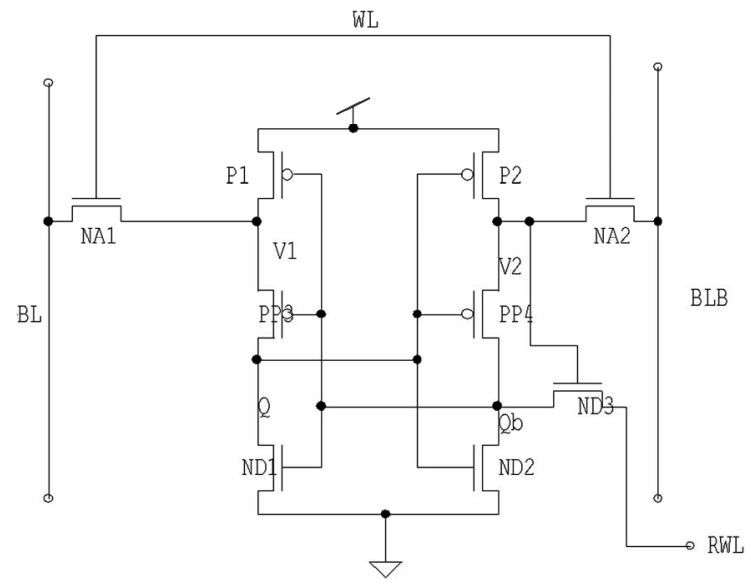

Figure 2. Proposed PP based 9T SRAM cell.

node Qb in a complementary manner. Transistors P1, PP3, and ND1 form a P-P-N inverter and P1, PP4, ND2 form another.

ND1 provides the read current path for discharging a bitline (BL) or its complementary (BLB), depending on the stored values of $\mathrm{Q}$ and $\mathrm{Qb}$, respectively. The source terminal of this transistor is connected to the VGND pin, which connects to the ground voltage only during the read operation. Anytime else, it stays high to curb unnecessary leakage current.V1 and V2 are located between the two cascaded P-MOS transistors forming the P-P-N inverter. Q and Qb are the storage nodes .BL and BLB are bitlines while $\mathrm{Wl}$ is the word line as in conventional 6T SRAM cell.

\subsection{Working of the Novel 9T Cell}

\subsubsection{Write Operation}

During a write operation .initially in this PP based 9T SRAM Figure 2, storage node Q stores " 0 ” while Qb stores " 1 ". To perform a write operation, the wordline WL is enabled and one bitline, e.g., BLB, is pulled down to ground in advance. When the supply voltage is relatively high (e.g., $1 \mathrm{~V}$ ), node Qb (storing "1") here in this case will be pulled down directly through the discharging path formed by. In turn, node Q will be charged up to complete the data-flipping process.

In general, the lower portion of our P-P-N inverter pair can be viewed as a latch consisting of PP3-ND1and PP4ND2. In some sense, this latch takes node V1and node V2 as the pseudo supply terminals. In step $1, \mathrm{Qb}$ is pulled down quickly to nearly the ground voltage at the beginning of the write operation since it is driven by BLB tied to strong "0". Qb via the PMOS between them (PP3), reducing the voltage of $\mathrm{Qb}$ to a lower middle voltage. During this time period, PP3 and PP4 controlled by Qb still conducts weakly to pull up voltage at node Q, Due to the coupling effect of parasitic capacitances the voltage of $\mathrm{Qb}$, which is in the floating state, rises with node Q but only slightly. In step 2, the data flipping finally takes place when $\mathrm{Q}$ is strong enough to conduct the PDR transistor to discharge $\mathrm{Qb}$ down to the ground voltage.

It is worth mentioning that even though such a write mechanism takes relatively longer time to accomplish the data flipping, it is still shorter than the read access time, and therefore, overall it does not introduce any operating frequency penalty. We can also further improve the cell's write-ability by strengthening the access transistors (NA1 and NA2). It does not affect the read performance.

\subsubsection{Read Operation}

To perform a read operation, the wordline WL is enabled and RWL is pulled down to ground to allow bitline discharging. Assuming that the data stored at Q is now " 0 ". Since data node Q and Qb are isolated from bitline BL by PP2 and PP3 (which is between the true storage node Q) and thus the so-called read current (which is the current used to discharge a bitline) does not flow through the storage node $\mathrm{Qb}$ but through the bypassing $N D 3$ as indicated in Figure 2. This is the main reason why the read stability does not degrade at all in our cell. As for a $6 \mathrm{~T}$ cell, the read current flows through the storage node directly, thereby causing read disturbance, i.e., the voltage at data node $\mathrm{Q}$ will rise temporarily. This will degrade the read stability because the cell flipping will be more likely to take place.

The pull-up transistors P1 and P2 are usually made weaker for easy write operation just like in a conventional 6T cell. While the pull-down transistors ND1 and ND2, forming the cell discharging paths, need to be stronger to facilitate a larger read current and thereby a quicker access. The pass gate transistors NA1 and NA2 need to be strong enough to serve as high-conduction paths between the accessed cell and the bitlines during both the read and write operations. The two pull-up transistors, PP2 and PP3, need to be slightly stronger, to compensate for the conductivity degradation of the cascaded PMOS structure linking the storage nodes (i.e., $\mathrm{Q}$ and $\mathrm{Qb}$ ) and Vdd, which help contribute to a good hold SNM. Unlike a $6 \mathrm{~T}$ cell, the pull-down transistors do not have to be strong, since they do not involve in the cell discharging paths. However, their strengths are made comparable to the cascaded PMOS structure mentioned above to achieve a more balanced cell structure which could lead to a larger hold SNM.

In our cell we have seen that the SVNM has been 1.53times greater than that of conventional $6 \mathrm{~T}$ cell. In our cell we achieved $460 \mathrm{mv}$ while for $6 \mathrm{~T}$ it is $300 \mathrm{mv}$. The leakage power has also been reduced by 3.3 times than that of conventional 6T. 


\section{Stability Measurement: N Curve Analysis}

Numerous analytical models of the static noise margin (SNM) have been developed to optimize the cell design, to predict the effect of parameter changes on the SNM and to assess the impact of intrinsic parameter variations on the cell stability. Furthermore, new SRAM cell circuit designs have been developed to maximize the cell stability for future technology nodes [8]. The set up for $\mathrm{N}$ curve is as shown in Figure 3. In an ideal case, each of the two cross-coupled inverters in the SRAM cell has an infinite gain. As a result, the butterfly curves delimit a maximal square side of maximum, being an asymptotical limit for the SNM. Therefore, scaling limits the stability of the cell. An additional drawback of the SNM is the inability to measure the SNM with automatic inline testers [4], due to the fact that after measuring the butterfly curves of the cell the static current noise margin (SINM) still has to be derived by mathematical manipulation of the measured data. An alternative definition for the SRAM read stability is based on the N-curve of the [5]. N-curve contains information both on the read stability and on the writeability, thus allowing a complete functional analysis of the SRAM cell with only one N-curve [8].

Parameters which are find by using $N$ curve these 4 parameters are useful for measuring the write ability and read ability of the cell.

- The static voltage noise margin (SVNM)

The static voltage noise margin is the voltage difference between points A and B in Figure $\mathbf{4}$ and it indicates the maximum tolerable DC noise voltage at the input of the inverter of the cell before its content changes [4].

- The static current noise margin (SINM)

The static current noise margin is defined as the maxi-

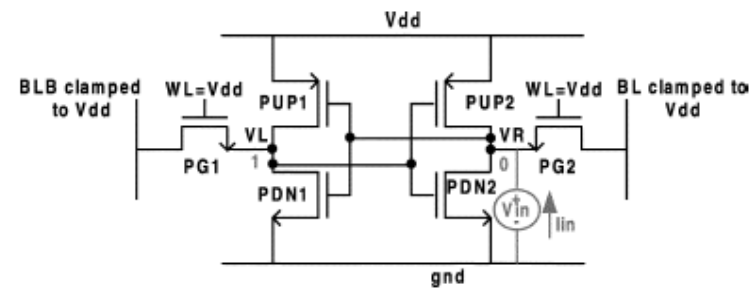

Figure 3. Set up for $\mathbf{N}$ curve analysis.

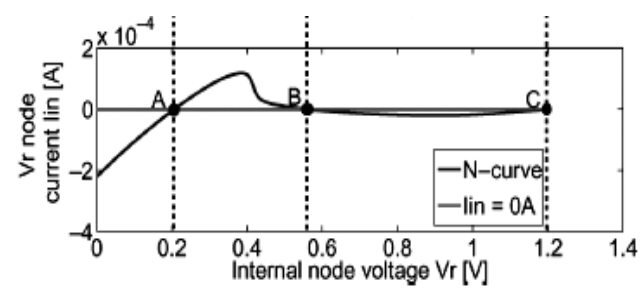

Figure 4. Ncurve for 6 T SRAM cell [2]. mum value of DC current that can be injected in the SRAM cell before its content changes [4]. It is given by the peak value of Iin during read operation that is between points A and B in the Figure 4.

- The Write Trip Voltage (WTV)

The SRAM N-curve also provides information regarding the write ability of the cell. WTV is the voltage drop needed to flip the internal node "1" of the cell with both the bit lines clamped at Vdd [4]. It is given by the voltage difference between the second (B) and the last zero crossing point (C) in Figure 4.

- The Write Trip Current (WTI)

It is the amount of current needed to write the cell when both bit lines are clamped at supply voltage equal to Vdd [5]. The peak value of Iin after the second zero crossing of N-curve gives WTI.

For better read stability, the values of SVNM, and the magnitude of SINM and hence the value of static power noise margin SPNM (product of mean of SVNM and mean of SINM) should be larger. For better write ability the value of WTV, the absolute value of WTI and hence the value of WTP (product of mean of WTV and mean of WTI) must be smaller.

\section{Analysis of N Curve Metrics}

$\mathrm{N}$-curve analysis has been done at $45 \mathrm{~nm}$ technology in order for low voltage operation. Various factors of stability has been analyses with the affect of temperature and voltage on them. Figure 5 shows one $\mathrm{N}$ curve analysis at $\mathrm{Vdd}=1 \mathrm{~V}$ at temperature varies from $-25^{\circ} \mathrm{C}$ to $125^{\circ} \mathrm{C}[2]$.

\subsection{Effect of Power Supply (Vdd)}

We have seen that there is significant affect of power supply on the 4 parameters which we have obtained from the Ncurve.As Vdd increases the stability also increases [9]. This is also been observed here that as the Vdd increased the SINM, WTV, SVNM and WTI the four parameters has been increased as shown in the given graphs.

\subsubsection{Effect on SVNM}

As shown in the graph Figure 6, we see that at Vdd $=1$ $\mathrm{V}$ it is maximum $460 \mathrm{mv}$ and reduces when we go to Vdd $=0.6 \mathrm{~V}$. As we know that SVNM it is the maximum DC tolerable voltage before the cell changes it contents so it means that as Vdd reduces the cell tolerance is also reduces.

\subsubsection{Effect on WTI}

As discussed above the write-trip current (WTI) is the 


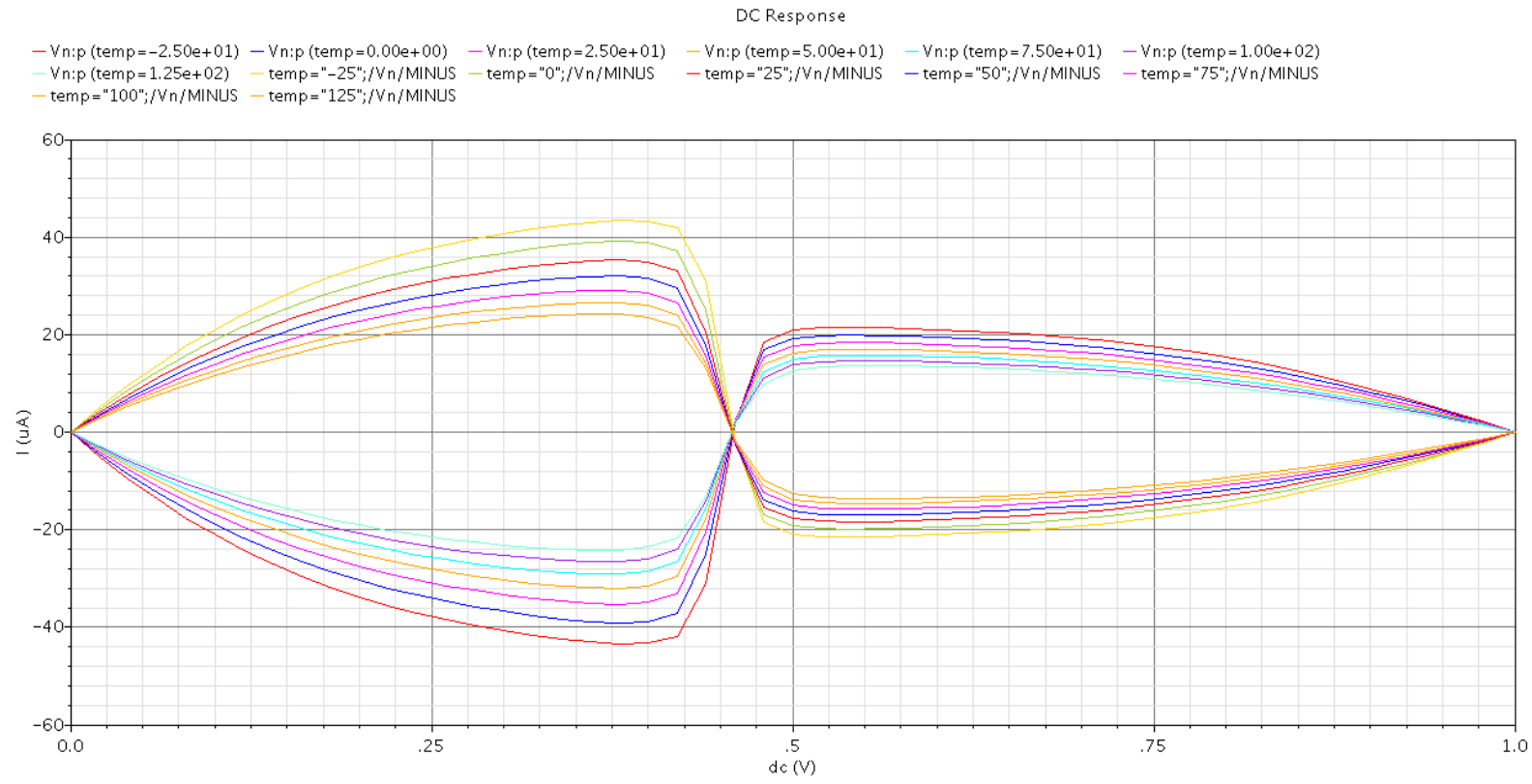

Figure 5. N curve of 9T SRAM cell.

amount of current needed to write the cell when both bit-lines are kept at Vdd . This is the current margin of the cell for which its content changes as in (Figure 7). The ability to write a cell with both bit-lines clamped at results actually in a destructive read operation; therefore, the absolute value of WTI should be large enough to cope with the read stability requirement. On the other hand, the lower the absolute WTI is, the higher the write-trip point of the cell. It shows an exponential relation with Vdd. WTI is measure at various temperatures.

\subsubsection{Effect on WTV}

Write-trip voltage (WTV) is the voltage drop needed to flip the internal node " 1 " of the cell with both the bit-lines clamped at Vdd. Write ability requires both WTI and WTV.WTV increases with Vdd. At $1 \mathrm{~V}$ the cell has maximum stability and it decreases drastically when the Vdd reaches to 0.6 V. As shown in Figure 8.

\subsubsection{Effect on SINM}

By using the combined SVNM and SINM, the read stability criteria for the cell are defined properly. For example, a small SVNM combined with a large SINM will still result in a stable cell since the amount of required noise charged disturb the cell is large. At Vdd $1 \mathrm{~V}$ we have good SINM but it reduces exponentially at $\mathrm{Vdd}=$ $0.6 \mathrm{~V}$, as shown in Figure 9.

\subsection{Effect of Temperature}

As we have varied the temperature from $0^{\circ} \mathrm{C}$ to $125^{\circ} \mathrm{C}$ we have seen that the SVNM and WTV is unaffected by the temperature variation but the currents i.e. the write trip current and static noise margin current has been affected by temperature variation. As temperature increases both the SINM and WTI reduces. As shown in Figures $\mathbf{1 0}$ and $\mathbf{1 1}$ respectively. The variation has been observed at varying Vdd from $1 \mathrm{~V}$ to $0.6 \mathrm{~V}$.

\section{Analysis of Leakage Current for Proposed Cell}

In this cell we have achieved 33\% less leakage power with respect to 6T SRAM cell, as in this cell we have used the PMOS cell and also we used ND3 which is used to reduced the leakage power. We have seen the affect of Vdd and temperature on leakage power. As we know it depends exponential to Temperature and increases with temperature the same affect is seen here Figure 12. It also shows the effect of Vdd which shows that there is $7 \mathrm{X}$ increases in Leakage current when we increase the Vdd from $0.6 \mathrm{~V}$ to Vdd $1 \mathrm{~V}$.

We have also analyzed the Leakage power with SINM as shown in Figure 13 and found that as SINM increases the Leakage power also increases which shows that with increasing Vdd the SINM increases and at the same time increasing Vdd results in increasing the leakage power [10].

\section{Conclusions}

We have proposed a novel 9T SRAM cell which has 


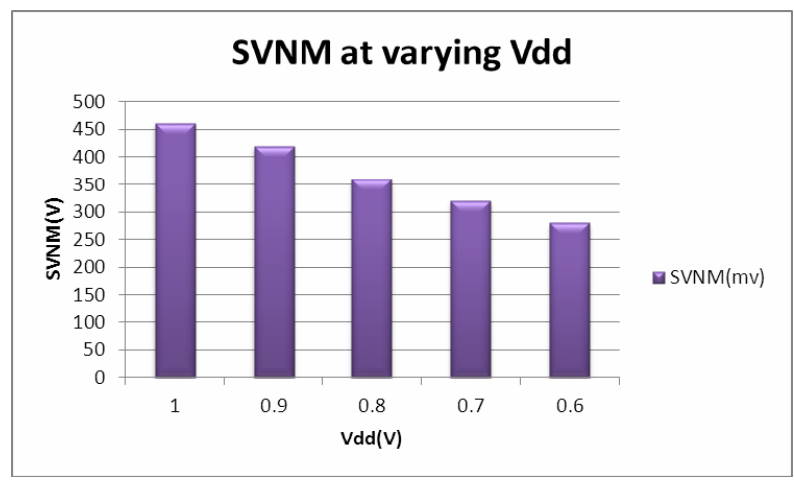

Figure 6. Vdd vs. SVNM.

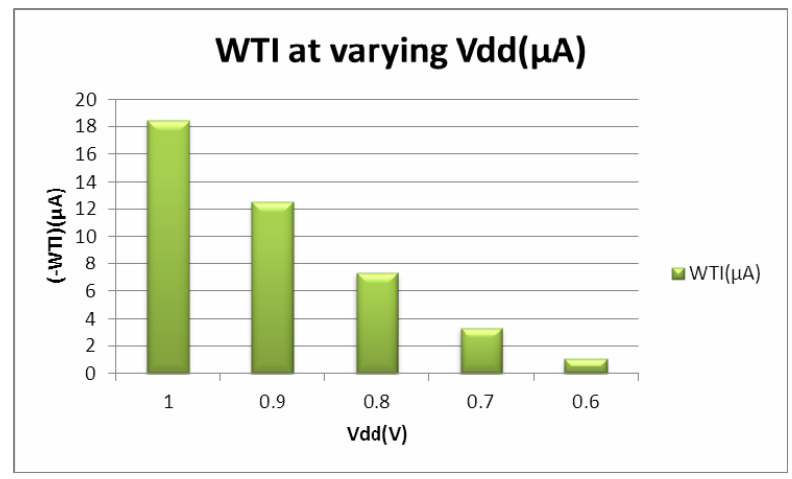

Figure 7. Vdd vs. WTI.

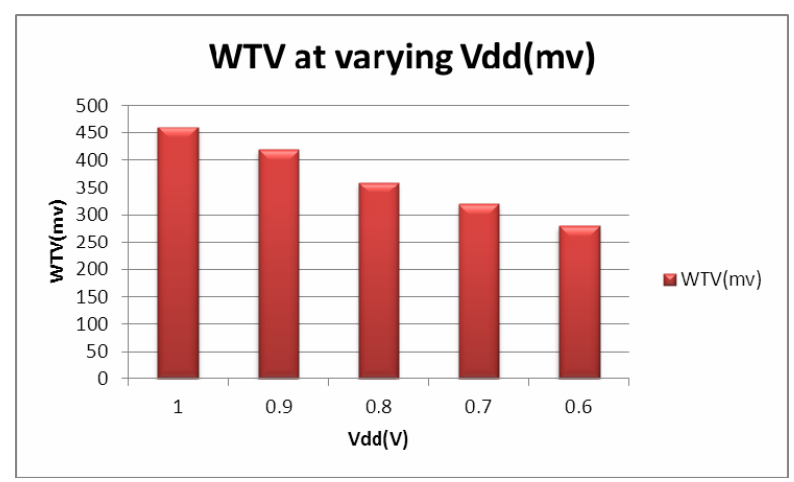

Figure 8. Vdd vs. WTV.

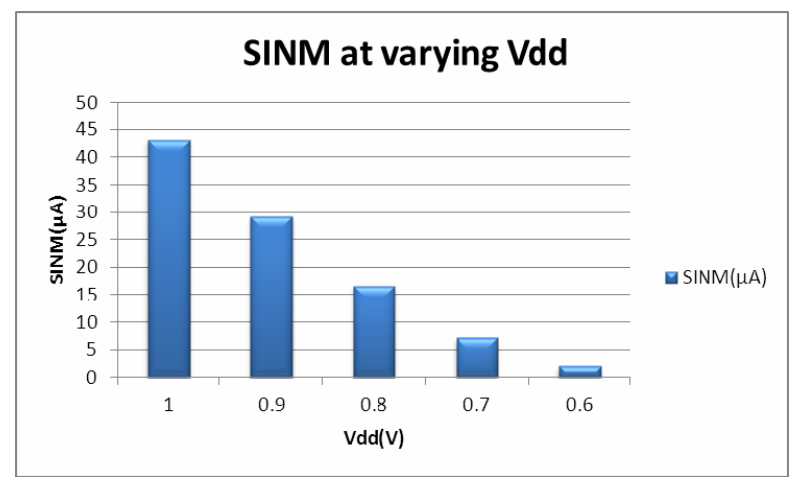

Figure 9. Vdd vs. SINM.

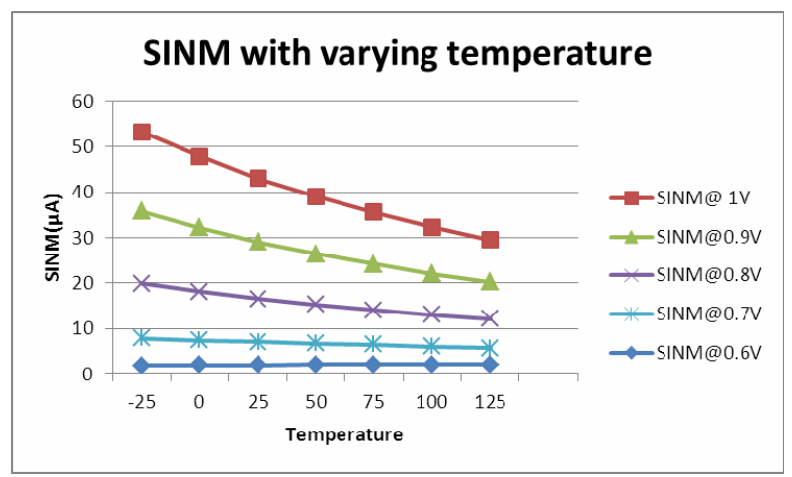

Figure 10. Temperature vs. SINM.

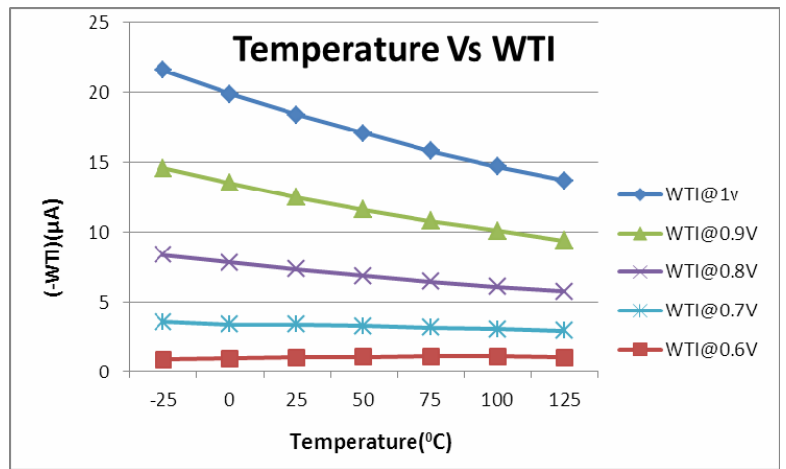

Figure 11. Temperature vs. WTI.

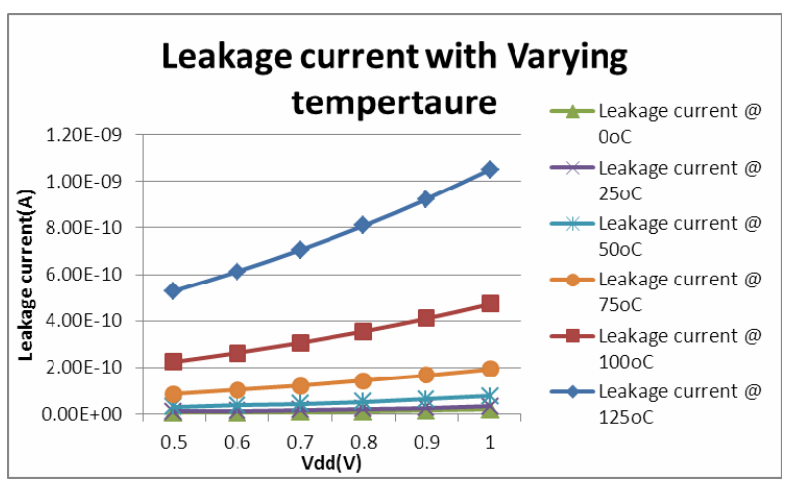

Figure 12. Temperature vs. leakage current.

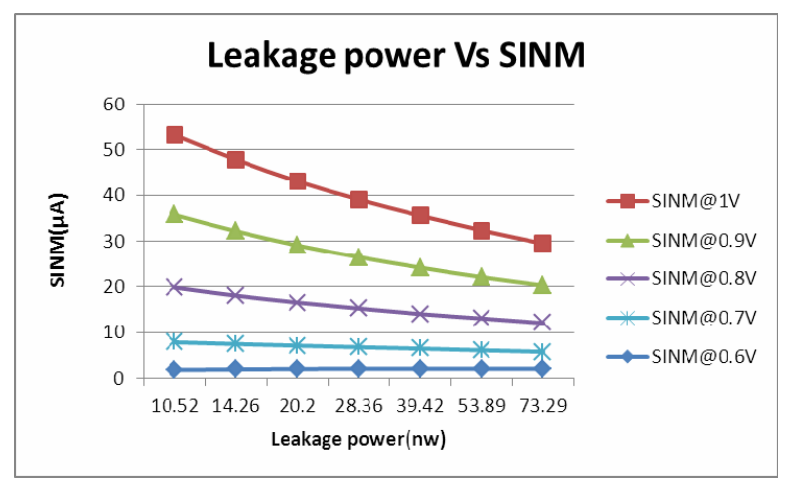

Figure 13. Leakage power vs. SINM. 
been simulated at $45 \mathrm{~nm} 65 \%$ increase in SVNM compared to 6T SRAM cell. The cell has 33\% leakage power reduction also with respect to 6T SRAM cell and in this we have not used any leakage reduction techniques. So the future expansion can be done by sizing the cell to increase the stability i.e. the write ability and read ability of the cell. Also power can be reduced by using various leakage reduction methods. Although the area with respect to $6 \mathrm{~T}$ has been increased but at lower technology it is comparable to $6 \mathrm{~T}$. The SNM measured is $380 \mathrm{mv}$ which can be improved by sizing the transistor widths.

\section{Acknowledgements}

The authors are very grateful to the respective organization for their support and encouragement.

\section{References}

[1] M. Sinangil, V. Naveen and A. P. Chandrakasan, "A Reconfigurable 8T Ultra-Dynamic Voltage Scalable (U-DVS) SRAM in $65 \mathrm{~nm}$ CMOS," IEEE Journal Solid-State of Circuits, Vol. 44, No. 11, 2009, pp. 3163-3173. doi:10.1109/JSSC.2009.2032493

[2] R. E. Aly and M. A. Bayoumi, "Low-Power Cache Design Using 7T SRAM Cell," IEEE Transactions on Circuits and Systems II: Express Briefs, Vol. 54, No. 4, 2007, pp. 318-322. doi:10.1109/TCSII.2006.877276

[3] Z. Liu and V. Kursun, "Characterization of a Novel Nine-Transistor SRAM Cell,” IEEE Transaction of Very large Scale Integration Systems, Vol. 16, No. 4, 2008, pp.
488-492.

[4] B. H. Calhoun and A. P. Chandrakasan "Static Noise Margin Variation for Sub-threshold SRAM in $65 \mathrm{~nm}$ CMOS,” IEEE Journal of Solid-State Circuits, Vol. 41, No. 7, 2006, pp. 1673-1679. doi:10.1109/JSSC.2006.873215

[5] Y. Chung and S.-H. Song, "Implementation of LowVoltage Static RAM with Enhanced Data Stability and Circuit Speed,” Microelectronics Journal, Vol. 40, No. 6, 2009, pp. 944-951. doi:10.1016/j.mejo.2008.11.063

[6] E. Seevinck, et al., "Static-Noise Margin Analysis of MOS SRAM Cells,” IEEE Journal of Solid-State Circuits, Vol. 22, No. 5, 1987, pp. 748-754. doi:10.1109/JSSC.1987.1052809

[7] C. Wann, et al., "SRAM Cell Design for Stability Methodology,” 2005 IEEE VLSI-TSA International Symposium on VLSI Technology, 25-27 April 2005, pp. 21-22.

[8] E. Grossar, M. Stucchi and K. Maex, "Read Stability and Write-Ability Analysis of SRAM Cells for Nanometer Technologies," IEEE Journal of Solid-State Circuits, Vol. 41, No. 11, 2006, pp. 2577-2588. doi:10.1109/JSSC.2006.883344

[9] S. Birla, M. Pattanaik and R. K. Singh, "Static Noise Margin Analysis of Various SRAM Topologies,” IACSIT International Journal of Engineering and Technology, Vol. 3, No. 3, 2011, pp. 304-309.

[10] S. Birla, N. Kr. Shukla, M. Pattanaik and R. K. Singh, "Device and Circuit Design Challenges for Low Leakage SRAM for Ultra Low Power Applications," Canadian Journal on Electrical \& Electronics Engineering, Vol. 1, No. 7, 2010, pp. 157-167. 